\title{
On The Feasibility of Polar Code as Channel Code Candidate for the 5G-IoT Scenarios
}

\author{
Arti Sharma* and Mohammad Salim \\ Department of Electronics and communication, MNIT, Jaipur, India \\ 2013rec9048@mnit.ac.in,msalim.ece@mnit.ac.in
}

\begin{abstract}
$5 G$ is on the horizon and internet of things (IoT) is anticipated as an eminent scenario for the $5 G$ network paradigm. The emerging 5G-IoT scenarios are required to potentially support and encourage the massive number of devices of low cost, low latency, long battery life with high reliability. IoT is envisioned to have billions of simultaneous connections over next few years that will completely change the characteristics of mobile network. M2M communication is prime enabling technology of the IoT which is much related to the 5G's Machine type Communication (MTC). Therefore, 5G's machine type Communication between massive numbers of devices with mission critical services is become very popular now days and named as 5G-IoT scenario. Channel Coding for the 5G-IoT scenarios is facing novel challenges as to support its various requirements on latency, reliability, scalability and energy consumption. Hence, an FEC code needs to endorse diverse requirements of code lengths and rates, as well as high throughput with low decoding complexity. This paper compares various candidate channel coding methods for 5G-IoT scenario. Polar code is believed as dominant advancement in channel coding theory and guarantees for apical performance. And hence, polar code is considered as promising candidate for the 5G-IoT scenario. This paper put an emphasis on the suitability of polar codes for 5G-IoT scenario.
\end{abstract}

Keywords: 5G; Internet of Things; Polar Code; Mission Critical MTC; mMTC

\section{Introduction}

Forthcoming fifth generation $(5 \mathrm{G})$ of mobile communication is on the horizon and expected to have initial commercialization by 2020 [1]. IoT is the prominent revolution in the next generation mobile ecosystems. IoT is an emerging concept where, almost all everyday "things"-physical objects (that have sensing, communicating and processing capability) can talk with other devices, anywhere and anytime, through the internet and/or telecommunication network [2]. In addition to mobile broadband (MBB) services, IoT has become key enabler for the 5G NR and gained immense attention of various industries and academics. MBB supports high data rate services while IoT is primarily driven by machine type communication that is further divided into categories based on nature of communication between devices/machines [5]: massive machine type communication (mMTC) and Mission-critical MTC (MC-MTC). The mMTC networks are expected to provide low data rate services to massive number of low cost and low energy (battery constrained) devices with sparse short information [17]. The MC-MTC networks require low latency and ultra high reliability [7]. Thus, 5G-IoT scenarios include the characteristics of both mMTC and MC-MTC use cases. In other words, 5G-IoT scenarios as depicted in Figure 1, are interpreted as 5G's machine type Communication between massive numbers of devices with mission critical services.

Received (November 25, 2017), Review Result (February 12, 2018), Accepted (March 28, 2018)

* Corresponding Author 


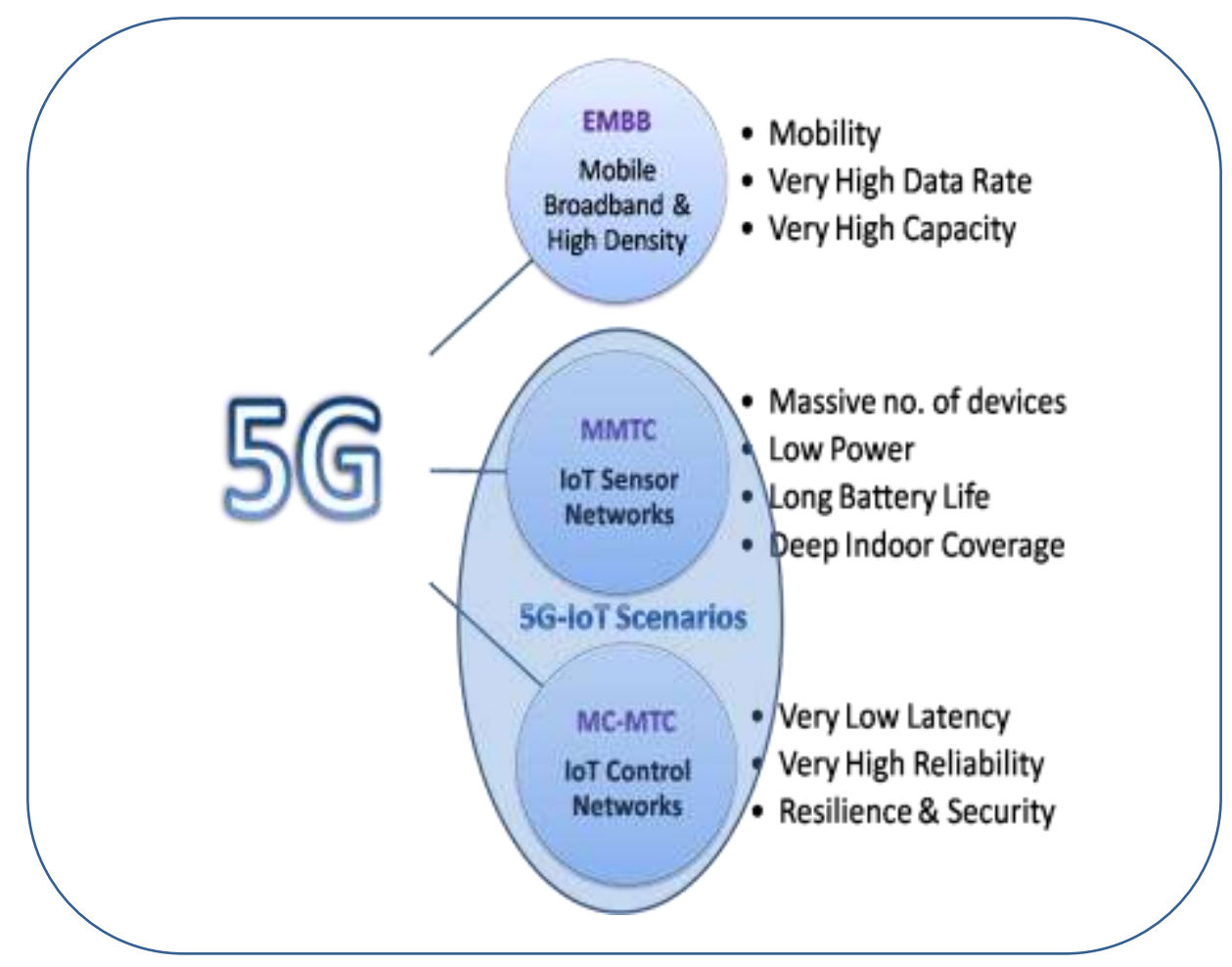

Figure 1. A Simple Depiction of 5G-IoT Scenarios

Channel coding plays an important role in New Radio Access Technology (NRAT) for the $5 \mathrm{G}$ as it needs to support various use scenarios and diverse set of applications [19]. Channel coding for 5G-IoT scenarios require variety in code rates and code block lengths with high throughput, high reliability, low latency and low decoding complexity (for low energy consumption) [6]. Channel coding schemes for existing LTE systems i.e. LTETurbo Code and LTE- TBCC (Tail- Biting Convoltutional Code) no longer support all new requirements for 5G NR [8]. LDPC codes show wonderful performance with large block length and high code rate but unfortunately, perform inadequately over low code rates $(<1 / 3)$ and short block lengths ( $<400$ bits) [18], which are typical for 5G-IoT. Polar code discovered by Erdal Arikan in 2009, is a new contender in this race. Polar code is a class of linear block code based on the concept of Channel polarization. Polar code has explicit code construction and simple decoding with modest complexity and memory requirements [11], which render them appealing for 5G NR scenarios such as battery constrained IoT applications [10]. Polar code with effortless methods for puncturing (variable code rates) and code shortening (variable code lengths) can achieve better performance than that of state-of-the art codes [14]. All these make polar code as an appropriate choice for $5 \mathrm{G}-\mathrm{IoT}$ scenarios.

This paper focuses on suitable channel coding schemes for 5G-IoT scenarios. All the channel coding candidates for MC-MTC and mMTC scenarios are compared.

This paper is organized as follows: section II presents the basics of the polar codes. Channel coding requirements for 5G-IoT scenarios are described in Section 3. Suitability of polar code is depicted in Section 4. Simulation results are compared for various coding methods in Section 5.

\section{Polar Code: Background}

Polar Code is a new class of linear block codes based on phenomena known as channel polarization. According to Arikan (2009) polar code is the first known code that provably achieves the Shannon's capacity for symmetric binary input discrete memory-less channels (B-DMC). Some inclusive benefits of polar codes such as explicit construction, 
modest encoding and decoding complexity with no error floor drawing attention of various industries and academia from past few years [10]. Three basic building blocks of a polar code are described as follows.

\subsection{Code Construction}

The code construction is based on channel polarization which transforms an ordinary channel $\mathrm{W}$ into two extreme channels for which Channel coding problem is trivial: i) Perfect or Good - A noiseless channel that transmits information without error (max. capacity $\mathrm{C}(\mathrm{W})=1$ ) known as reliable channel. ii) Useless or bad - An extremely noisy channel that transmits only random noise $(\mathrm{C}(\mathrm{W})=1)$ known as Unreliable channel.

Preliminary: Consider a B-DMC W: $\mathrm{X} \rightarrow \mathrm{Y}$ with Input alphabet $\mathrm{X}=\{0,1\}$, arbitrary output alphabet $Y$ and their transition probability $\{\mathrm{W}(\mathrm{y} / \mathrm{x}), \mathrm{x} \in \mathrm{X}, \mathrm{y} \in \mathrm{Y}\} . \mathrm{W}^{\mathrm{N}}$ denote $\mathrm{N}$ independent copies of $\mathrm{W}$. Mutual Information of $\mathrm{W}$ (B-DMC) between $\mathrm{X}$ and $\mathrm{Y}$ is expressed as

$I(W)=\sum_{y \in Y} \sum_{x \in x_{2}^{-}} W(y / x) \log \frac{w(y / x)}{\frac{1}{2} w(y / 0)+\frac{1}{2} w(y / 1)}$

Assuming $\mathrm{W}$ as a symmetric channel then its capacity is equal to $\mathrm{I}(\mathrm{W})$. Bhattacharyya Parameter $\mathrm{Z}(\mathrm{W})$ defines reliability of a channel, given by

$$
\mathrm{Z}(\mathrm{W})=\sum_{\mathrm{y} \in \mathrm{Y}} \sqrt{W(\mathrm{y} / 0) W(\mathrm{y} / 1)}
$$

\section{Channel Polarization}

$\mathrm{N}$ Independent copies of $\mathrm{B}-\mathrm{DMC}, \mathrm{W}$ are combined recursively to produces a vector channel $\mathrm{W}_{\mathrm{N}}: \mathrm{X}^{\mathrm{N}} \rightarrow \mathrm{Y}^{\mathrm{N}}$, where $\mathrm{N}=2^{\mathrm{n}} ; \mathrm{n} \geq 0$, and then split back into $\mathrm{N}$ transformed copies of channel $\left\{W^{i}: 1 \leq \mathrm{i} \leq \mathrm{N}\right\}$ such that the new parallel channels are polarized. That is the symmetric capacity of new channels reaches to either 1 or 0 , when $\mathrm{N}$ becomes large. After obtaining such polarized channels, polar coding transmits only $\mathrm{K}$ information bits over $\mathrm{W}(\mathrm{i})$ channels which are reliable and transmit frozen bits (no information i.e., send bit = 0 ) over the remaining $\mathrm{N}-\mathrm{K}$ channels. Example of basic transformation for channel combining is shown in Figure 2 where two channels are combined to produce a new channel $\mathrm{W}_{2}$ with inputs $\mathrm{u}_{1}, \mathrm{u}_{2}$ and outputs $\mathrm{y}_{1}, \mathrm{y}_{2}$ and transition probabilities

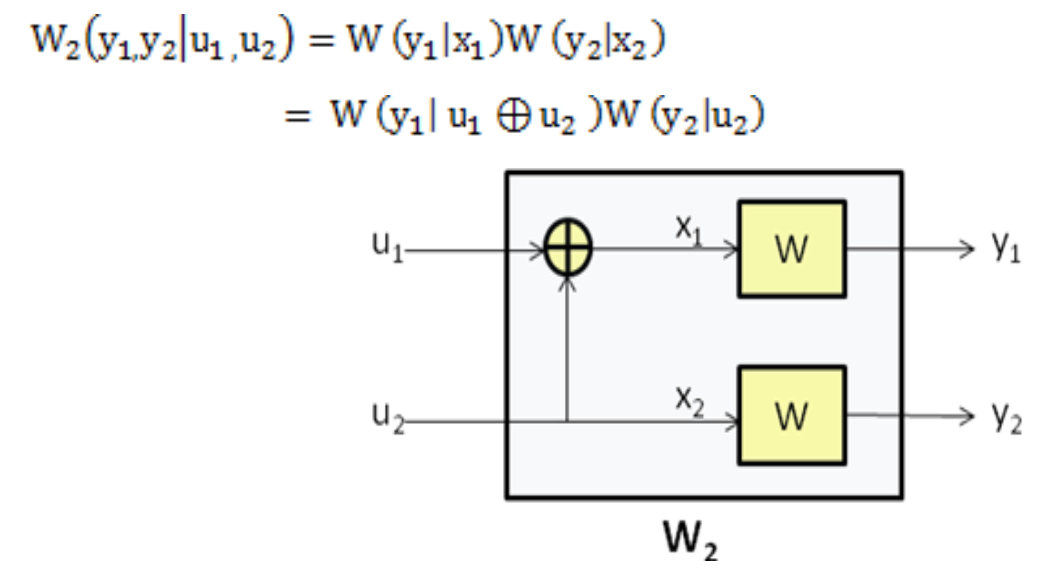

Figure 2. Example of Channel Polarization (Combing)

There is one-to-one mapping (Linear transformation) between $\left(\mathrm{U}_{1}, \mathrm{U}_{2}\right)$ and $\left(\mathrm{X}_{1}, \mathrm{X}_{2}\right)$. Polar coding combines two same copies of $\mathrm{W}_{2}$ to frame new channel $\mathrm{W}_{4}$ and two copies of $\mathrm{W}_{4}$ to produce $\mathrm{W}_{8}$ so on. After that, Vector channel $\mathrm{W}_{\mathrm{N}}$ is split back to generate $\mathrm{N}$ polarized channels. 


$$
\left.\mathrm{W}_{N}^{(\mathrm{i})}:\{0,1\} \rightarrow \mathrm{y}^{\mathrm{N}} \times\{0,1\}^{\mathrm{i}-1}, 1 \leq \mathrm{i} \leq \mathrm{N}\right\}
$$

Transition probability of channels after splitting is,

$$
\mathrm{W}_{\mathrm{N}}^{(\mathrm{i})}\left(\mathrm{y}_{1}^{\mathrm{N}}, \mathrm{u}_{1}^{\mathrm{i}-1} \mid \mathrm{u}_{\mathrm{i}}\right)=\frac{1}{2^{\mathrm{N}-1}} \sum_{\mathrm{u}_{\mathrm{i}+1}^{\mathrm{N}}} \mathrm{e}(0,1)^{\mathrm{N}-\mathrm{i}} \mathrm{W}_{\mathrm{N}}\left(\mathrm{y}_{1}^{\mathrm{N}} \mid \mathrm{u}_{1}^{\mathrm{N}}\right)
$$

\subsection{Polar Encoder}

A polar code $(\mathrm{N}, \mathrm{K})$ with block length $\mathrm{N}$ and $\mathrm{K}$ information bits has generation matrix $G_{N}=B_{N} F^{\otimes n}$.

Where, $\mathrm{B}_{\mathrm{N}}$ is the bit-reversal permutation matrix. $\mathbf{F}^{\otimes n}$ is the $\mathrm{n}$-th order Kronecker product of $\mathrm{F}$,

$$
F=\left[\begin{array}{ll}
1 & 0 \\
1 & 1
\end{array}\right]
$$

Let a vector $x_{1}^{N}$ of length $N$, denotes output of encoder, computed from

$$
x_{1}^{N}=u_{1}^{N} G_{N}
$$

Where, $u_{1}^{N}=u_{1}, \ldots \ldots . u_{N}$ denote data sequence, split into two sets i) indices corresponding to information bits to be sent on the good channels (Set A); ii) remaining indices related to frozen bits to be transmitted on the bad channels $\left(\operatorname{Set} \mathrm{A}^{\mathrm{C}}\right.$ ). Thus, equation 8 can be written as

$$
\mathrm{x}_{1}^{\mathrm{N}}=\mathrm{u}_{\mathrm{A}} \mathrm{G}_{\mathrm{N}}(\mathrm{A}) \oplus \mathrm{u}_{\mathrm{A}^{\mathrm{c}}} \mathrm{G}_{\mathrm{N}}\left(\mathrm{A}^{\mathrm{c}}\right)
$$

\subsection{Decoder}

Arikan found that [9] Successive cancellation (SC) decoder is fundamental polar decoder for achieving capacity with moderate complexity. SC decoder successively estimates $\hat{u}_{1}^{N}$ of input sequence $u_{1}^{N}$ from received sequence $y_{1}^{N}$. For each $u_{i}$ decoder take $\mathrm{N}$ decisions, now i) if $\mathrm{u}_{\mathrm{i}}$ is a frozen bit then decoder sets $\widehat{\mathrm{u}}_{\mathrm{i}}=0$, ii) if $\mathrm{u}_{\mathrm{i}}$ is information bit, calculate likelihood ratio (LR) once estimating all previous bits $u_{1}^{i-1}$.

$$
\begin{aligned}
& \mathrm{L}_{\mathrm{N}}^{(\mathrm{i})}\left(\mathrm{y}_{1}^{\mathrm{N}}, \widehat{\mathrm{u}}_{1}^{\mathrm{i}-1}\right)=\frac{\mathrm{w}_{\mathrm{N}}^{\mathrm{G}}\left(\mathrm{y}_{1}^{\mathrm{N}}, \mathrm{u}_{1}^{i}-1 \mid 0\right)}{\mathrm{w}_{\mathrm{N}}^{(\mathrm{i})}\left(\mathrm{y}_{1}^{\mathrm{N}}, \mathrm{u}_{1}^{i-1} \mid 1\right)} \\
& \hat{\mathrm{u}}_{\mathrm{i}}= \begin{cases}0, & \text { if } \mathrm{L}_{\mathrm{N}}^{(\mathrm{i})}\left(\mathrm{y}_{1}^{\mathrm{N}}, \hat{\mathrm{u}}_{1}^{\mathrm{i}-1}\right) \geq 1 \\
1, & \text { Otherwise. }\end{cases}
\end{aligned}
$$

Polar code construction for $\mathrm{N}=8$ is shown in Figure 3. The numbers on left side represents capacities of eight channels corresponding to the eight bits. The bits with high capacities are information bits and remaining are frozen bits. Here, $\mathrm{K}=4$ and Code rate $\mathrm{R}=1 / 2$.

Several modifications in the basic SC decoding algorithm have been proposed to improve finite-length performance of polar code. SC List (SCL) decoding [12] uses L simultaneous decoding paths for apparent performance improvement, even comparable to that of MAP decoding. According to Huawei white paper [3] polar code when concatenated with high rate CRC codes (CRC-aided SC List CA-SCL) can perform better than state-of-art codes (LDPC and Turbo codes). SC decoder with low memory and complexity is still an open issue to solve. 


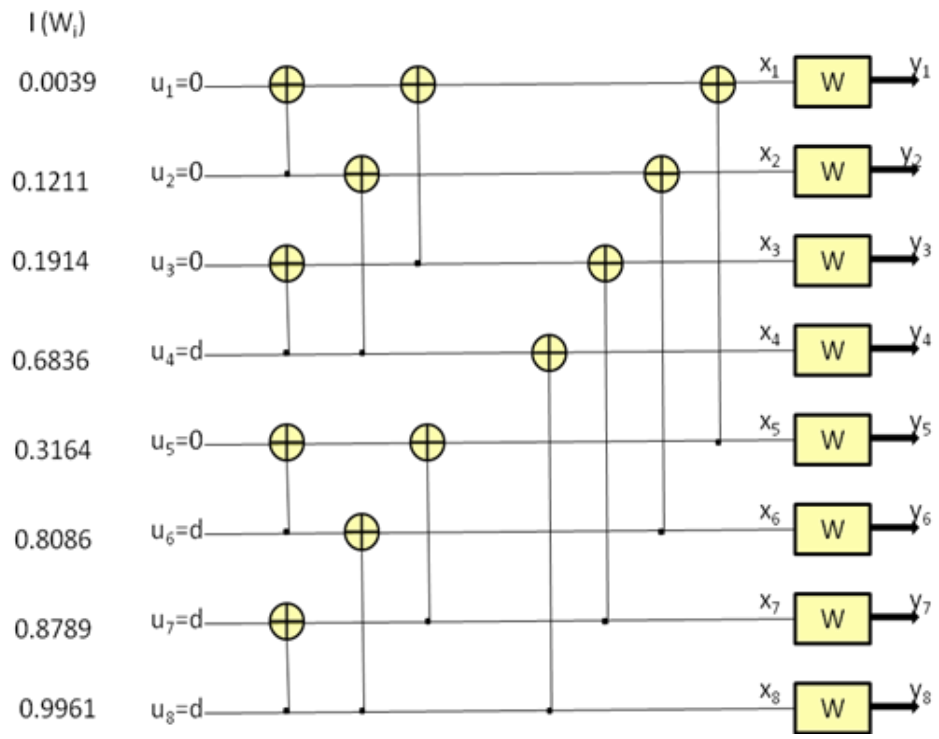

Figure 3. Schematic for Polar Coding

\section{Potential Channel Coding Requirements for 5G-IOT Scenarios}

Over next few years, 5G and IoT will endorse innumerable emerging use cases and applications with miscellaneous performance aspects [4]. Concerning this, ITU-R in September 2015 agreed upon its vision for IMT-2020 and beyond networks. The most challenging requirement for 5G-IoT scenario is to support massive number of devices [2]. For this, the cost of a terminal should be significantly low. The energy consumption has to be low enough that the device battery-life backing for years without recharging. Also, there should be seamless network coverage even in basements [5].

The main characteristic of IoT communication is transmission of Small packets which is often associated with low SNR [6]. According to Huawei white paper [3], the advanced channel coding schemes can confer robust error protection in extreme coverage scenarios. For low cost and low power consumption, the channel coding methods can use low complexity encoding and decoding algorithms [8]. For this, the channel coding schemes can optimize code structure (block length, code rate, encoding and decoding structure) to provide desired performance. In case of short block, CRC bit reduction can be an impressive approach for overhead reduction [6]. This approach can be used for both turbo and polar codes. This section briefly explains major requirements of 5G-IoT (MC-MTC and mMTC) scenarios and maps those requirements on channel coding methods.

\subsection{Mission Critical MTC Scenario}

MC-MTC use case imposes harsh requirements on latency and reliability [4]. This use case is very similar to 5G URLLC (Ultra reliable low latency communication) scenario with short information. General KPI requirements for URLLC scenario are described in [5]. i) Reliability can be measured in terms of block error rate of $10^{-5}$ with or without HARQ support during 1-ms period. ii) Target latency is $1 \mathrm{~ms}$ in general $(0.5 \mathrm{~ms}$ for uplink transmission and $0.5 \mathrm{~ms}$ for downlink). When these requirements are mapped on the channel coding schemes then we need a code that should have characteristics shown in Figure 4.

Optimizing channel coding schemes for such reliability and latency requires a paradigm shift in state-of-the art coding methods [18]. Finding the channel coding scheme for such use case is an open problem. 3GPP has already started its contribution towards this. 


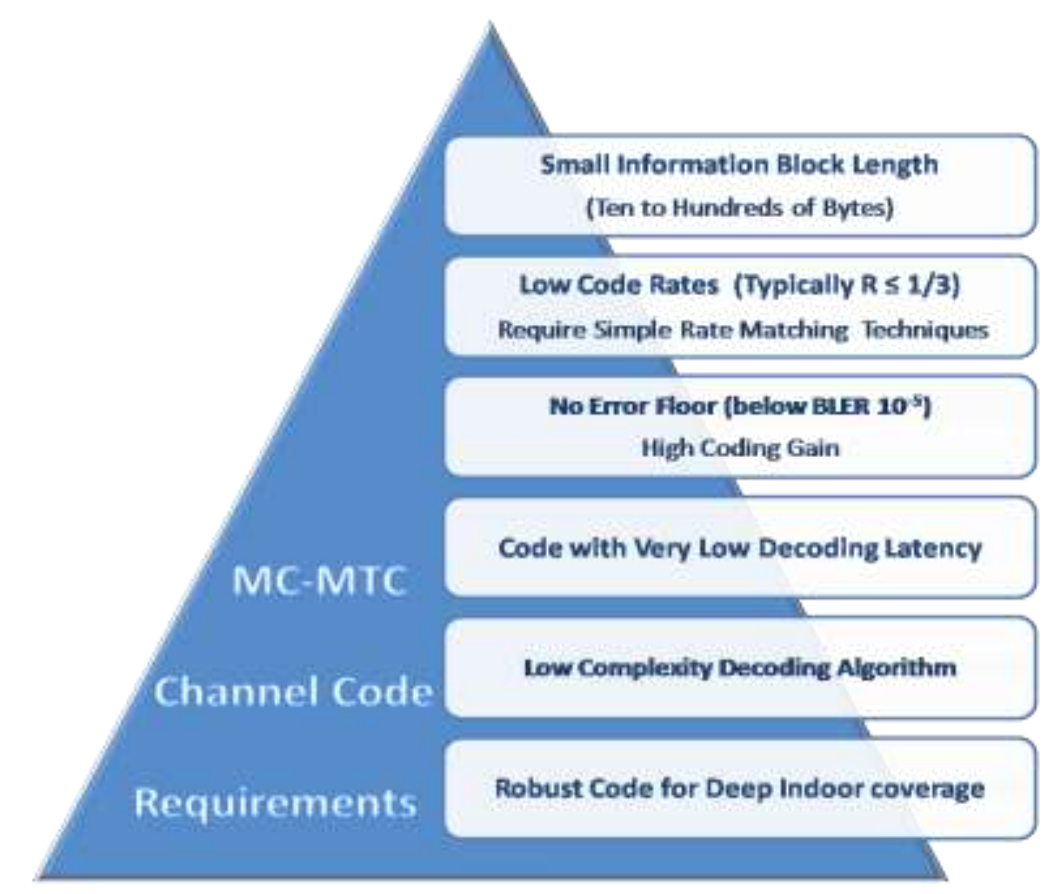

Figure 4. Channel Coding Requirements for MC-MTC Scenario

\section{2. mMTC Scenario}

The Massive machine type communications i.e., mMTC scenario endows wireless connectivity to billions of low complexity and low energy (battery constrained) devices [17]. These applications require transmission of relatively small sized and non-delaysensitive information. Both 3GPP and IEEE have already started working towards standardization of requirements for $5 \mathrm{G} \mathrm{mMTC}$ scenario [4]. The key requirements for this scenario are the use of lower-order modulation schemes with shorter block size information to satisfy low power requirements. Therefore, following discussion is mainly based on 20- 200 info block sizes with QPSK modulation scheme [6].

The channel coding scheme for mMTC scenario needs to support a code with i) shorter block lengths (tens to hundreds of bytes), ii) lower-order modulation (QPSK) iii) energyefficient and low complexity encoding and decoding which is essential for massive longlife devices [18].

\section{Suitability of Polar Code for 5G- IOT Scenarios}

At first, in October 2016 a Chinese firm Huawei used Polar codes as channel coding method in 5G field trials and achieved downlink speed of 27Gbps [13]. After this in November 2016, $3 \mathrm{GPP}$ standardized polar code as dominant coding for control channel functions in 5G eMBB scenario in RAN 86 and 87 meetings. The channel coding methods for the URLLC (or MC-MTC) and mMTC scenarios have not yet been agreed and the candidates are Turbo codes, TBCC, LDPC codes and Polar codes [18]. While considering requirements for these two scenarios, turbo code is no more in the race due to presence of error floor which make it inadmissible for highly reliable communication. High complexity iterative decoding results in low throughput, high latency, more energy and memory requirements. Also, miserable performance for shorter block lengths and low code rates makes turbo code unfit for 5G-IoT scenario. LDPC codes shows inferior performance for short block lengths $(<400$ bits $)$ and low code rates $(<1 / 3)$ which is typical scenario for MC-MTC and mMTC use cases. In case of TBCC codes, no further improvements have been observed towards $5 \mathrm{G}$ requirements. 
Polar Code is consider to be the strongest contender for the MC-MTC and mMTC use cases as it offers variety in code rates and code lengths (offer simple puncturing and code shortening mechanism respectively) with excellent performance. Polar codes have no error floor and thus they support $99.999 \%$ reliability which is mandatory for the ultra-high reliability requirements of 5G-IoT. Use of simple encoding and low complexity SC-based decoding lowers terminal power consumption in polar codes (20 times lower than turbo code for same complexity). Therefore, the battery life of devices boots enormously for IoT applications which demand for ultra-low power consumption. Polar code has lower SNR requirements than the other codes for equivalent error rate and hence, provides higher coding gain and increased spectral efficiency. Furthermore, CRC-less parity check codes desired for 5G-IoT scenarios rather than CRC aided polar codes. Universality, flexibility and versatility render polar code attractive for 5G-IoT scenarios.

\section{Simulation Results}

This paper evaluates the performance in terms of the Block Error Rate (BLER) vs. SNR. Here, authors are introducing all coding candidates for the MC-MTC and mMTC scenarios and compare them for different code rates and block lengths.

\section{Polar Code}

CRC-less Parity Check polar code (Wang, 2016) is used for low latency considerations. SCL decoding algorithm with list size 8 is employed for similar complexity consideration. A novel puncturing scheme is applied (Wang, 2014).

\section{Simulation Parameters}

Table 1. Simulation Assumptions for MC-MTC and MMTC Scenarios

\begin{tabular}{|c|c|c|c|c|}
\hline Parameters & \multicolumn{4}{|c|}{ Specifications } \\
\hline Channel model & \multicolumn{4}{|c|}{ AWGN } \\
\hline Modulation & \multicolumn{4}{|c|}{ QPSK } \\
\hline Code rate & \multicolumn{4}{|c|}{$1 / 12,1 / 6,1 / 3$} \\
\hline Info. Block length(bits) & \multicolumn{4}{|c|}{$20,40,200,600$} \\
\hline Coding scheme & Turbo & TBCC & LDPC & Polar \\
\hline Decoding Algorithm & $\begin{array}{c}\text { Max-log-map } \\
(\mathrm{SF}=0.75, \text { iteration }=8)\end{array}$ & List-1 Viterbi & $\begin{array}{c}\text { Min-sum } \\
\text { (20 iterations) }\end{array}$ & $\begin{array}{l}\text { CRC-less } \\
\text { SCL, L }=8\end{array}$ \\
\hline
\end{tabular}

Figure 4 proves the reliability of polar code desired for MC-MTC scenario. And, it is clear that there is no error floor appearing for BLER lower than $10^{-5}$.

All the channel coding candidates for 5G-IoT scenarios are compared in Figures 5, 6, 7 and 8 . These comparisons are made only for small block lengths that have been agreed in (3GPP TS 38.913, 2016) for URLLC and mMTC use cases. It is evident from the results that the polar code outperforms all the coding candidates for all code rates and block lengths taken into. 


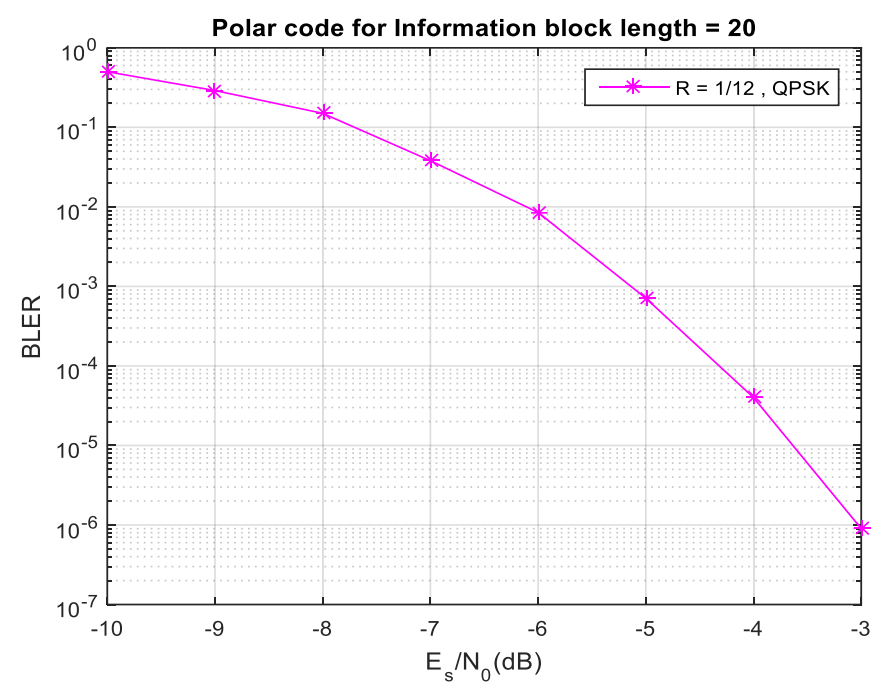

Figure 4. BLER Performance of Polar Code for MC- MTC Scenario

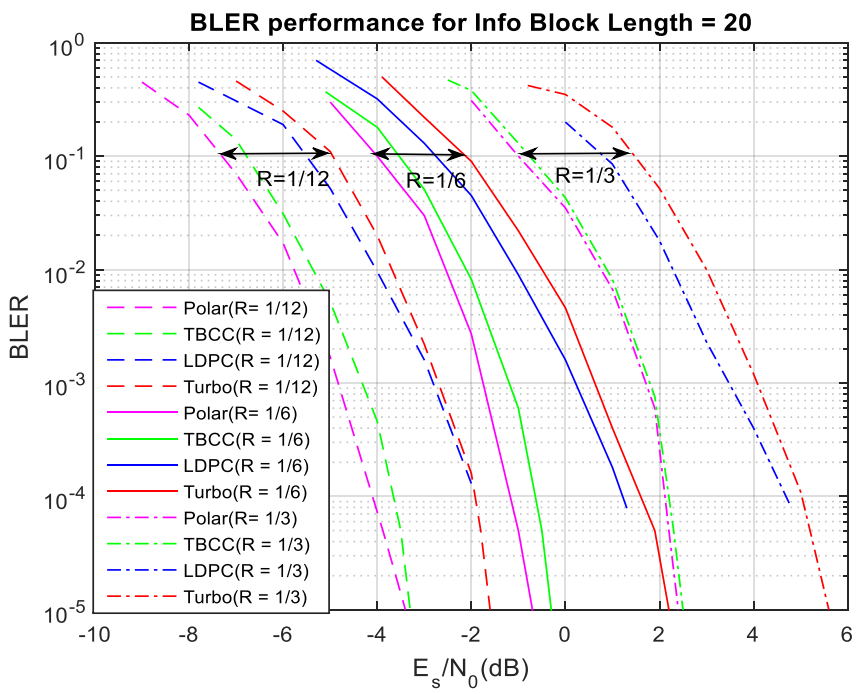

Figure 5. Comparison of Coding Schemes for $\mathrm{K}=20$

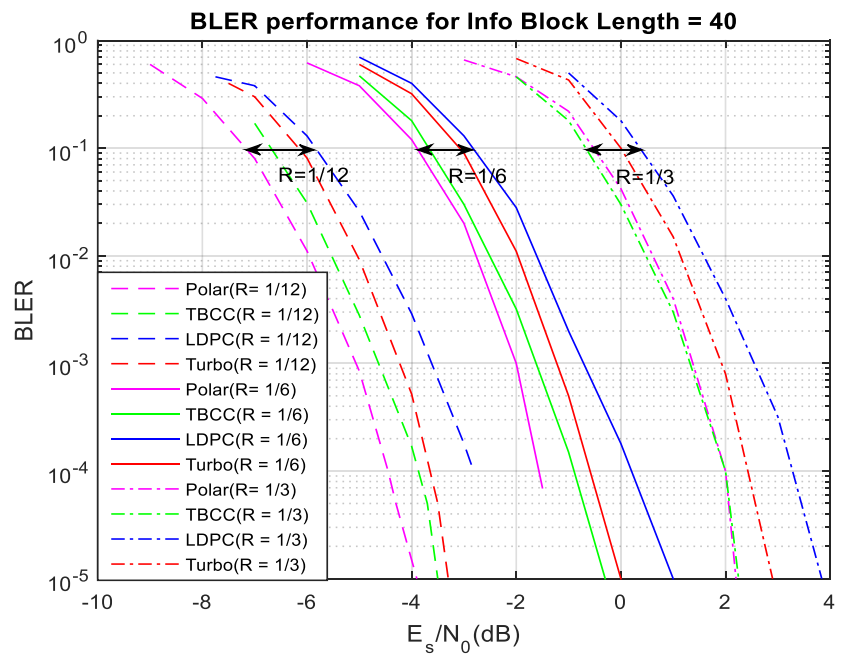

Figure 6. Comparison of Coding Schemes for $\mathrm{K}=\mathbf{4 0}$ 


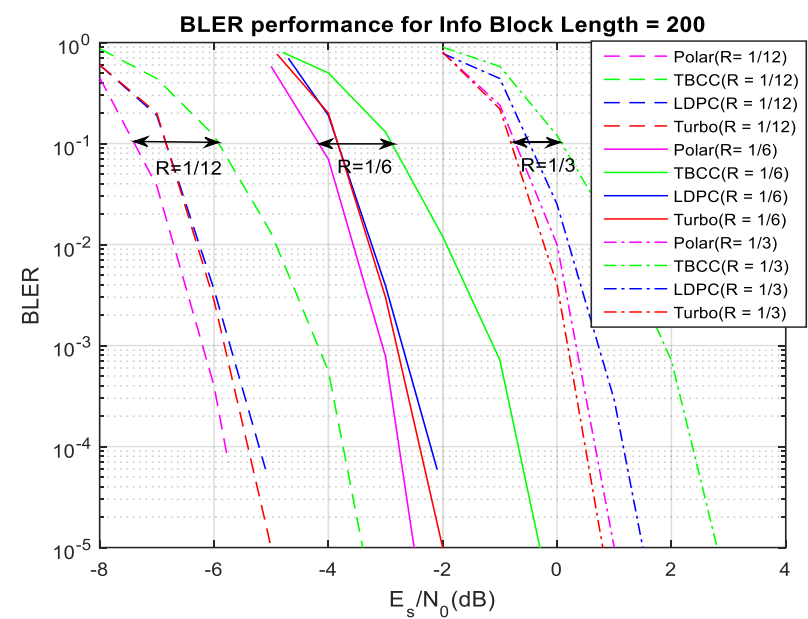

Figure 7. Comparison of Coding Schemes for $K=200$

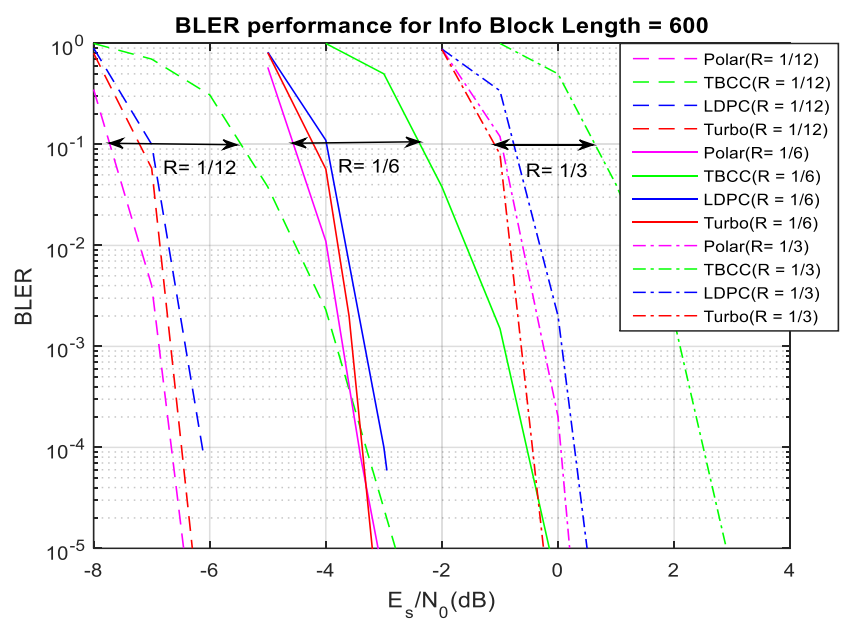

Figure 8. Comparison of Coding Schemes for K = 600

\section{Conclusion}

5G-IoT scenario demands for a paramount technology for channel coding purpose as to encourage various new applications. Polar code is considered to be a simplest and most efficient solution. This paper analyzes various channel coding candidates for 5G-IoT scenarios (MC-MTC and mMTC) especially for small block lengths and low code rates. It is observed that the polar code meets all KPI requirements of 5G-IoT scenarios. It is apparent from simulation results that the polar code outperforms other candidate codes for almost all considered block lengths and code rates. Polar code has excellent error correcting performance with no error floor observation. Therefore, Polar code is shown to be an obvious choice for lower code rates and smaller block lengths which desired for 5GIoT scenarios.

\section{Future Scope}

IoT systems integrated with the 5G are still an open area of research. Although polar code has been established and proven their potentiality, betterment in performance of low complexity decoders for small block lengths is a subject to analyze. To support mission critical massive number of devices, implementation cost, latency and practicability of polar codes (with respect to state- of- art codes) are some issues to explore. Therefore, 
Channel coding for $5 \mathrm{G}$ is an active investigation area as to face many outstanding challenges in coming years.

\section{References}

[1] P. Demestichas, A. Georgakopoulos and J. Lu, "5G on the horizon: key challenges in radio access network", IEEE vehicular technology magazine, vol. 8, no. 3, (2013), pp. 47-53.

[2] M. Rita Palattella, M. Dohler and A. Grieco, "Internet of Things in the 5G Era: Enablers, Architecture, and Business Models", IEEE Journal on Selected Areas in Communications, vol. 34, no. 3, (2016), pp. 510-527.

[3] Huawei Whitepaper, "New air interface and radio access virtualization", (2015).

[4] M. Shafi, A. F. Molisch and P. J. Smith, "5G: A Tutorial Overview of Standards, Trials, Challenges, Deployment and Practice", IEEE journals on Selected Areas in Comm., vol. 35, no. 99, (2017), pp. 1201-1220.

[5] Z. Qi and F. HP, "Mission critical IoT communication in 5G", Edited Atanasovski V., Leon-Garcia A., Future Access Enablers for Ubiquitous and Intelligent Infrastructures, Springer, Cham, vol. 159, (2015), pp. 35-41.

[6] G. Durisi, T. Koch and P. Popovski, "Towards massive, ultra-reliable, and low-latency wireless: The art of sending short packets", Proc. IEEE, vol. 104, no. 9, (2016), pp. 1711-1726.

[7] M. Sybis, K. Wesolowski and K. Jayasinghe, "Channel Coding for Ultra-Reliable Low Latency Communication in 5G Systems", IEEE Vehicular Technology Conference, Montreal, Canada, (2016) September, pp. 1-6.

[8] I. Onurcan, L. Diego and X. Wen, "A Comparison of Channel Coding Schemes for 5G Short Message Transmission", IEEE Globecom workshop, (2016) October, pp. 1-6.

[9] Arıkan, "Channel polarization: A method for constructing capacity achieving codes for symmetric binary-input memoryless channels", IEEE Trans. Inf. Theory, vol. 55, no. 7, (2009), pp. 3051-3073.

[10] A. Erdal, "Challenges and some new directions in channel coding", Journal of Communications and Networks, vol. 17, no. 4, (2015), pp. 328-338.

[11] I. Tal and A. Vardy, "How to construct polar codes", IEEE Trans. Inf. Theory, vol. 59, (2013), pp. $6562-$ 6582.

[12] I. Tal and A. Vardy, "List decoding of polar codes", IEEE Trans. Inf. Theory, vol. 61, no. 5, (2015), pp. 2213-2226.

[13] Retrieved 2016-10-10, "Huawei achieves 27Gbps 5G speeds with Polar Code".

[14] T. Wang, D. Qu and T. Jiang, "Parity-Check-Concatenated Polar Codes", IEEE Communications Letters, vol. 20, no. 12, (2016), pp. 2342-2345.

[15] R. Wang and R. Liu, "A novel puncturing scheme for polar codes", IEEE Communications Letter, vol. 18, no. 12, (2014), pp. 2081-2084.

[16] B. W. Khoueiry and M. Reza Soleymani, "A Novel Machine-to-Machine Communication Strategy Using Rateless Coding for the Internet of Things", IEEE Internet of Things Journal. vol. 3, no. 6, (2016), pp. 937-950.

[17] B. Carsten, N. Pratas and P. Popovski, "Massive Machine-type Communications in 5G: Physical and MAC-layer solutions", IEEE Communication magazine, vol. 54, no. 9, (2016), pp. 59-65.

[18] 3GPP TSG RAN WG1, R1-167703, "Channel coding scheme for URLLC, mMTC and control channels", Intel Corporation, (2016).

[19] 3GPP TS 38.913, "Study on scenarios and requirements for next generation access technologies", (2016). 